\title{
Tratamiento ambulatorio de la diverticulitis aguda no complicada. Análisis de nuestra experiencia
}

\author{
Outpatient treatment of uncomplicated acute diverticulitis. Analysis of our experience
}

\author{
Andrés Garcia-Marín*, Lorena Rodríguez-Cazalla, Cristina Bernabeu-Herráiz, Beatriz Madrid-Baños, \\ Miguel Morales-Calderón y Antonio Compañ-Rosique \\ Servicio de Cirugía General y del Aparato Digestivo, Hospital Universitario San Juan de Alicante, Alicante, España
}

\begin{abstract}
Resumen
Objetivo: Evaluar la seguridad y la eficacia del tratamiento con antibiótico oral para la diverticulitis aguda no complicada, y realizar un análisis de costos. Método: Estudio prospectivo, no aleatorizado, entre junio de 2014 y junio de 2017. Se incluyeron todos los pacientes diagnosticados de diverticulitis aguda no complicada según la clínica y la tomografía abdominal en el servicio de urgencias del Hospital Universitario San Juan de Alicante (España). El tratamiento ambulatorio consistió en antibiótico oral durante 7 días (amoxicilina-ácido clavulánico o ciprofloxacino y metronidazol en alérgicos a los betalactámicos), dieta líquida durante 72 horas y analgésicos. Los costos fueron evaluados según la Ley de Tasas de la Comunidad Valenciana. Resultados: Se incluyeron 90 pacientes, 49 mujeres y 41 hombres, con una mediana de edad de 56 años. La tasa de éxito fue del 95.5\% ( $n=86)$, necesitando ingreso hospitalario cuatro pacientes $(4.5 \%)$. El tratamiento antibiótico empleado fue amoxicilina-ácido clavulánico en 82 pacientes (91.1\%) y ciprofloxacino con metronidazol en ocho pacientes (8.9\%). El ahorro por paciente fue de 1985 euros en comparación con el hipotético ingreso de todos los pacientes. Conclusiones: El tratamiento ambulatorio de la diverticulitis aguda no complicada se completó con éxito en la mayoría de los pacientes, permitiendo una importante reducción del gasto.
\end{abstract}

PALABRAS CLAVE: Diverticulitis aguda no complicada. Tratamiento ambulatorio. Infección intraabdominal.

\begin{abstract}
Objective: Analyze the safety and efficacy of the outpatient treatment of uncomplicated acute diverticulitis and a costs analysis. Method: We conducted a prospective, non-randomized study between June 2014 and June 2017. We included all patients diagnosed of uncomplicated acute diverticulitis based on clinical and abdominal computed tomography scan in the Emergency Department of the University Hospital San Juan de Alicante (Spain). Outpatient treatment consisted of oral antibiotics for 7 days (amoxicillin-clavulanate or ciprofloxacin and metronidazole in patients with betalactamic allergy), liquid diet for $72 h$ and analgesics. Costs were evaluated according to the Law of Rates of Valencian Community. Results: Ninety patients were included, 49 females and 41 males with a median age of 56 years. Success rate was $95.5 \%(n=86)$ requiring hospital admission 4 patients (4.5\%). Antibiotic treatment was amoxicillin-clavulanate in 82 patients (91.1\%) and ciprofloxacin and metronidazole in 8 (8.9\%). Cost savings per patient was approximately $1985 €$ comparing with hypothetically all inpatient treatment. Conclusions: Outpatient treatment of uncomplicated acute diverticulitis can be performed successfully in most patients allowing an important cost savings.
\end{abstract}

KEY WORDS: Uncomplicated diverticulitis. Outpatient treatment. Intraabdominal infection.

\author{
Correspondencia: \\ *Andrés García Marín \\ Carretera Nacional, 332 - Alicante-Valencia, s/n Fecha de recepción: 28-04-2018 \\ C.P. 03550, San Juan de Alicante (Alicante), España Fecha de aceptación: 11-07-2018 \\ E-mail: andres.garciam @ goumh.umh.es \\ DOI: $10.24875 / C I R U .18000408$
}

Cir Cir. 2019;87:40-44

Contents available at PubMed www.cirugiaycirujanos.com 


\section{Introducción}

La diverticulitis aguda es la complicación más frecuente de la enfermedad diverticular, y es uno de los motivos de consulta más comunes en los servicios de urgencias. El tratamiento ambulatorio con dieta líquida y antibioticoterapia ha sido defendido por varios estudios para el manejo de la diverticulitis aguda no complicada (DANC), frente al ingreso hospitalario ${ }^{1-12}$.

Los objetivos de nuestro estudio fueron evaluar la seguridad y la eficacia del tratamiento con antibiótico por vía oral para la DANC, y realizar un análisis de costos.

\section{Método}

Estudio prospectivo, analítico, de una serie de pacientes con diagnóstico de DANC en el servicio de cirugía de urgencias del Hospital Universitario de San Juan de Alicante (España) durante un periodo de 3 años (de junio de 2014 a junio de 2017), con una población adscrita de 240.000 habitantes.

Se incluyeron aquellos pacientes con diagnóstico de DANC de acuerdo con la clasificación radiológica por tomografía computarizada (TC) de Ambrosetti (diverticulitis leve: engrosamiento de la pared del colon e inflamación de la grasa pericólica; diverticulitis grave: presencia de un absceso, aire o contraste extraluminal) que cumplieron los siguientes criterios: edad menor de 80 años, inmunocompetencia, ausencia de criterios de sepsis grave, ausencia de vómitos y aceptación por parte del paciente y sus familiares ${ }^{13}$.

La sepsis grave se define como aquella sepsis (síndrome de respuesta inflamatoria asociada a una infección documentada por la clínica o microbiológicamente) con disfunción de órganos, hipotensión o hipoperfusión tisular. El síndrome de respuesta inflamatoria sistémica se define por el cumplimiento de al menos dos de los siguientes criterios: frecuencia cardíaca $>90$ latidos por minuto, frecuencia respiratoria $>20$ respiraciones por minuto, temperatura $>380<$ $36{ }^{\circ} \mathrm{C}$, leucocitos $>12,000 / \mathrm{mm}^{3} \mathrm{o}<4000 / \mathrm{mm}^{3}$. Las variables de disfunción orgánica son: renal, creatinina $>2 \mathrm{mg} / \mathrm{dl}$; hepática, plaquetas $<100,000 / \mathrm{mm}^{3}$; bilirrubina total, $>2 \mathrm{mg} / \mathrm{dl}$; y alteración de la coagulación con International Normalized Ratio (INR) > 1.50 Tiempo de tromboplastina parcial activado (TTPA) > 60 segundos). Las variables de hipotensión arterial son: presión sistólica $<90 \mathrm{mmHg}$ o descenso de esta > $40 \mathrm{mmHg}$ con respecto a su basal en ausencia de
Tabla 1. Coste del tratamiento de la diverticulitis aguda.

\begin{tabular}{lc}
\hline Tratamiento ambulatorio (completo) & Euros \\
\hline Urgencia hospitalaria & 189.49 \\
Antibiótico & 6.81 \\
Primera consulta de revisión (4. ${ }^{\circ}$ día) & 65.02 \\
Hemograma + proteína C reactiva & 9.76 \\
Consulta sucesiva (revisión al mes) & 40.02 \\
Total & 311.1 \\
\hline Tratamiento hospitalario (reingreso) & 2333.07 \\
\hline GRD 183 & 65.02 \\
Primera visita de revisión & 189.49 \\
Tasa urgencia hospitalaria (primera atención) & 2587.6 \\
\hline Total
\end{tabular}

otras causas de hipotensión. La variable de hipoperfusión tisular es: lactato $>2 \mathrm{mmol} /$ litro $^{14}$.

El protocolo de tratamiento ambulatorio consistió en antibiótico oral durante 7 días (amoxicilina-ácido clavulánico, $875 \mathrm{mg}$ cada 8 horas; en alérgicos a los betalactámicos, ciprofloxacino, $500 \mathrm{mg}$ cada 12 horas, más metronidazol, $500 \mathrm{mg}$ cada 8 horas), dieta líquida, analgesia y revisión clínica y analítica (hemograma y proteína $\mathrm{C}$ reactiva) al cuarto día, progresando a una dieta baja en fibra si la tolerancia era adecuada y revisión al mes, solicitando en los casos en que proceda una colonoscopia. El fracaso del tratamiento se definió por la necesidad de ingreso hospitalario en el primer mes tras el diagnóstico inicial y tratamiento ambulatorio.

El estudio de los costes se basó en la Ley de Tasas de la Comunidad Valenciana, según la cual la tarifa por el tratamiento ambulatorio se obtiene de la sumatoria de los costes individuales de la tasa de urgencia hospitalaria, la antibioticoterapia, la primera consulta al cuarto día con hemograma y proteína $C$ reactiva, y la sucesiva consulta al mes, mientras que la tarifa por proceso hospitalario se obtiene a partir de los Grupos Relacionados por Diagnóstico (GRD), que encuadran a la diverticulitis en el GRD 183, con independencia del plazo transcurrido entre el momento del ingreso y el alta hospitalaria, incluyendo la atención en urgencias, debiéndose añadir esta en el caso de un reingreso tras haber iniciado el tratamiento ambulatorio (Tabla 1)

La estadística descriptiva se realizó con frecuencia y porcentaje para las variables cualitativas, y con mediana y rango (valor mínimo-máximo) para las cuantitativas. 
Tabla 2. Resultados del laboratorio en el servicio de urgencias y en el control posterior.

\begin{tabular}{lcc}
\hline Parámetro & Analítica inicial & Analítica control (4. ${ }^{\circ}$ día) \\
\hline Leucocitos $/ \mathrm{mm}^{3}$ & $11,300(4200-21,400)$ & $6700(3000-12,500)$ \\
Neutrófilos (\%) & $74.1(45.7-85.1)$ & $54.5(31.2-76.8)$ \\
Linfocitos (\%) & $16.3(2.5-43.5)$ & $31.7(8.6-57.6)$ \\
$\begin{array}{l}\text { Proteína C } \\
\text { reactiva (mg/dl) }\end{array}$ & $4.95(0.06-22.6)$ & $1.43(0.05-20.9)$ \\
\hline
\end{tabular}

\section{Resultados}

De los 185 casos de diverticulitis aguda diagnosticados, se excluyeron 95 (30 por no cumplir todos los criterios del protocolo de tratamiento ambulatorio y 65 por corresponder a una diverticulitis complicada). De los 90 casos incluidos, 49 eran mujeres $(54.4 \%)$ y 41 eran varones (45.6\%), con una mediana de edad de 56 años (rango: $26-75)$. Un $56.7 \%$ de los pacientes $(n=51)$ tenían alguna enfermedad, siendo las más frecuentes la hipertensión arterial $(23.3 \%, \mathrm{n}=21)$ y el asma/bronquitis crónica $(7.8 \%, n=7)$. Un $30 \%(n=27)$ habían tenido un episodio previo de diverticulitis aguda, de tipo no complicado en 21 (80.8\%), complicado en 4 (15.4\%) y de ambos en 1 (3.8\%). El tiempo de evolución de los síntomas fue de 24 horas (rango: 10-336). Las localizaciones fueron el sigma $(72.2 \%, n=65)$, el colon descendente $(25.6 \%, n=23)$ y el colon derecho $(2.2 \%, n=2)$.

El tratamiento antibiótico empleado fue amoxicilina-ácido clavulánico en 82 pacientes (91.1\%), de los cuales presentaron diarrea $21(25.5 \%)$, y ciprofloxacino con metronidazol en 8 (8.9\%), sin aparición de diarrea.

El estudio comparativo de los resultados del laboratorio en el servicio de urgencias y en el control realizado en consultas al cuarto día se muestra en la tabla 2.

La tasa de reingreso fue del $4.5 \%(n=4)$ (Tabla 3), se realizó una nueva TC en todos los pacientes excepto en el que presentó vómitos, y no se objetivó empeoramiento de la diverticulitis. La evolución durante el ingreso fue correcta en todos ellos, sin modificar el tratamiento antibiótico. Un paciente que ingresó 8 días fue debido a una decisión del servicio de cirugía para cumplir el tratamiento antibiótico intravenoso completo, debido a la pérdida del apoyo familiar inicial.

El costo del tratamiento ambulatorio fue de 37,105 euros (86 pacientes con tratamiento ambulatorio completo y 4 pacientes con reingreso hospitalario), mientras que el costo de un hipotético tratamiento hospitalario de todos los casos hubiese sido de 215,828 euros, lo que supone una reducción del coste global del $82.8 \%$ y de 1985 euros por paciente durante el periodo de estudio.

\section{Discusión}

La diverticulitis aguda es una patología frecuente en nuestro servicio de urgencias, con un promedio de cinco casos mensuales, y la tasa de tratamiento ambulatorio es aceptable (75\% de las DANC), con un promedio de 2.5 casos mensuales.

En todos los estudios, con excepción del de Ridgway, et al. ${ }^{16}$, se realiza el diagnóstico de diverticulitis aguda mediante la asociación de la clínica, la analítica y el estudio radiológico, lo cual es básico en la actualidad para una adecuada caracterización de la patología y su inclusión en los diferentes estudios. La prueba de imagen empleada en todos los estudios, incluido el nuestro y con excepción del de Mizuki, et al.12, es la TC abdominal debido a su mayor sensibilidad y especificidad que la ecografía, ya que la caracterización de la gravedad ha de ser lo más correcta posible y la ecografía presenta su mayor problema en su dependencia del operador, la posible obesidad del paciente y la interposición de gas colónico ${ }^{3-12,16}$.

El criterio de inclusión de la mayoría de los estudios es la DANC ${ }^{7-9,11,17}$; sin embargo, algunos también incluyen la diverticulitis aguda con absceso pericólico de tamaño inferior a 2-3 cm (Tabla 4) $)^{5,10,12}$. Joliat, et al..$^{18}$ incluyen pacientes con absceso pericólico de tamaño inferior a $4 \mathrm{~cm}$ o neumoperitoneo local menor de $2 \mathrm{~cm}$, con una buena tasa de éxito. En nuestra serie solo hemos incluido pacientes con DANC debido a la necesidad de conseguir una alta tasa de éxito por parte de nuestro servicio de cirugía antes de plantearnos incluir los casos complicados leves de esta patología con una incidencia creciente.

El tratamiento básico consiste en dieta líquida durante las primeras 48-72 horas, analgesia y antibióticos, los cuales deben presentar una adecuada cobertura para bacilos gramnegativos y anaerobios. Los más empleados son la amoxicilina-ácido clavulánico o la combinación de ciprofloxacino y metronidazol, bien como elección o bien como alternativa en alérgicos a los

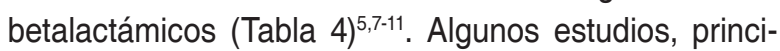
palmente de los primeros publicados, emplearon una dosis de antibiótico intravenoso durante la estancia en el servicio de urgencias ${ }^{10,17}$. El uso del tratamiento antibiótico en la DANC no está basado en la evidencia, de tal forma que dos ensayos clínicos han demostrado que este no acelera la recuperación ni previene las complicaciones y las recurrencias, por lo que su uso debería reservarse para la diverticulitis aguda complicada, situación que se encuentra en fases muy iniciales en nuestro medio y, por tanto, se requieren más estudios ${ }^{19-23}$. La 
Tabla 3. Características de los pacientes que reingresaron.

\begin{tabular}{|c|c|c|c|c|c|c|c|}
\hline Sexo & Edad & $\begin{array}{l}\text { Antecedentes } \\
\text { personales }\end{array}$ & $\begin{array}{l}\text { Diverticulitis aguda } \\
\text { recurrente }\end{array}$ & $\begin{array}{l}\text { Motivo de } \\
\text { ingreso }\end{array}$ & Leucocitos $/ \mathrm{mm}^{3}$ & $\begin{array}{l}\text { Segmento del } \\
\text { colon }\end{array}$ & $\begin{array}{l}\text { Días de } \\
\text { estancia }\end{array}$ \\
\hline Mujer & 73 & Depresión, Parkinson & Sí & Vómitos & 9700 & $\begin{array}{l}\text { Colon } \\
\text { izquierdo }\end{array}$ & 6 \\
\hline Mujer & 80 & Hipertensión arterial & Sí & Fiebre & 11,700 & Sigma & 8 \\
\hline Varón & 56 & No & No & $\begin{array}{l}\text { Aumento del } \\
\text { dolor }\end{array}$ & 5000 & $\begin{array}{l}\text { Colon } \\
\text { izquierdo }\end{array}$ & 2 \\
\hline Mujer & 51 & Hipertensión arterial & Sí & $\begin{array}{l}\text { Aumento del } \\
\text { dolor }\end{array}$ & 10,000 & Sigma & 3 \\
\hline
\end{tabular}

Tabla 4. Características de los principales estudios del tratamiento ambulatorio de la diverticulitis aguda no complicada.

\begin{tabular}{|c|c|c|c|c|c|}
\hline Autores & País (año publicación) & Gravedad & Antibiótico & Duración (días) & Reingreso \\
\hline Peláez, et al. ${ }^{11}$ & España, 2006 & DANC & AMX-CLAV/CIP+MTZ & 7 & $5 \%$ \\
\hline Ridgway, et al. ${ }^{16}$ & Canadá, 2009 & DANC & $\mathrm{CIP}+\mathrm{MTZ}$ & 7 & $0 \%$ \\
\hline Rodríguez-Cerrillo, et al. ${ }^{17}$ & España, 2010 & DANC & $\mathrm{CIP}+\mathrm{MTZ}$ & & $0 \%$ \\
\hline Moya, et al. ${ }^{9}$ & España, 2010 & DANC & $\mathrm{CIP}+\mathrm{MTZ}$ & 10 & $6.25 \%$ \\
\hline Lorente, et al. ${ }^{8}$ & España, 2013 & DANC & AMX-CLAV/CIP+MTZ & 7 & $5.6 \%$ \\
\hline Biondo, et al. ${ }^{7}$ & España, 2014 & DANC & AMX-CLAV/CIP+MTZ & 10 & $4.6 \%$ \\
\hline Mizuki, et al. ${ }^{12}$ & Japón, 2005 & Absceso $<2 \mathrm{~cm}$ & Cefpodoxima & 7 & $2.9 \%$ \\
\hline Martín-Gil, et al. ${ }^{10}$ & España, 2009 & Absceso $<3 \mathrm{~cm}$ & $\mathrm{CIP}+\mathrm{MTZ}$ & 7 & $5.4 \%$ \\
\hline Moya, et al. ${ }^{5}$ & España, 2016 & Absceso $<3 \mathrm{~cm}$ & $\mathrm{CIP}+\mathrm{MTZ}$ & 10 & $8.04 \%$ \\
\hline
\end{tabular}

AMX-CLAV: amoxicilina-ácido clavulánico; CIP + MTZ: ciprofloxacino + metronidazol; DANC: diverticulitis aguda no complicada.

duración habitual del tratamiento oscila, según los au-

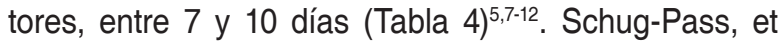
al. ${ }^{24}$ realizaron un estudio en el que compararon un tratamiento intravenoso de corta duración (4 días) frente a uno de 7 días, con similar tasa de éxito.

La tasa de reingreso en las series publicadas oscila entre el 0 y el $10 \%$, al igual que en nuestra serie, en la cual la causa más frecuente fue el aumento del dolor abdominal (Tabla 4). Etzioni, et al. ${ }^{25}$ determinaron que el sexo femenino y la presencia de líquido libre en la TC abdominal son factores de riesgo de fracaso del tratamiento ambulatorio ${ }^{5}$. Joliat, et al. ${ }^{18}$ encontraron como factores la admisión en el servicio de urgencias entre las 24:00 y la 6:00 horas, el neumoperitoneo local en la TC abdominal y una puntuación de Ambrosetti de 4. El principal problema para un estudio adecuado de los factores predictores de fracaso radica en el bajo tamaño muestral de las diferentes series. Así, Balasubramanian, et al. ${ }^{3}$, en su revisión sistemática de 10 estudios, hallaron que el fracaso ocurrió en 33 de 533 pacientes (6.2\%). Desde un punto de vista práctico, la mayoría de los autores, al igual que en nuestra serie, realizan un control clínico y analítico a los 3-4 días, en el cual debería objetivarse una mejoría clínica ${ }^{1,5,9}$. En nuestra serie hemos observado una disminución general de los parámetros inflamatorios en la analítica de control; sin embargo, no pudimos hacer un estudio de los factores predictores debido al escaso número de pacientes en los que fracasó el tratamiento ambulatorio.

En los países europeos, la experiencia con el tratamiento ambulatorio es principalmente en la diverticulitis aguda de colon descendente y sigma, descartando algunos estudios la diverticulitis de colon derecho $^{21}$. Sin embargo, Mizuki, et al. ${ }^{12}$, y Park, et al. ${ }^{26}$, han demostrado, debido a la mayor prevalencia de esta en los países asiáticos, que el tratamiento ambulatorio en estos casos también es factible. En nuestra serie hay dos casos de diverticulitis de colon derecho, cuyo tratamiento ambulatorio fue cumplido de forma correcta.

Los estudios de costos realizados por algunos autores han mostrado, al igual que en nuestra serie, una reducción del gasto asociado al proceso asistencial $(40-80 \%)$ y por paciente $(690-1870 \text { euros })^{3,7-10,12,17}$.

La principal debilidad radica en el tamaño muestral para poder obtener conclusiones en relación con el fracaso del tratamiento ambulatorio, siendo necesario para ello un estudio multicéntrico. 
Como nuevos proyectos de investigación en la patología estamos realizando un estudio comparativo en la DANC entre amoxicilina-ácido clavulánico y ciprofloxacino con metronidazol, valorando sus efectos adversos, y con posterioridad plantearemos un estudio comparativo entre un ciclo corto de antibióticos (4 días) y el clásico de 7 días.

\section{Conclusiones}

El tratamiento con antibiótico por vía oral de la DANC tiene una elevada tasa de éxito, con una baja tasa de reingresos (4.5\%), siendo el motivo más frecuente el aumento del dolor abdominal. La diarrea fue el efecto adverso más frecuente del tratamiento, asociado al uso de amoxicilina-ácido clavulánico, si bien sería necesario un estudio comparativo frente a la combinación de ciprofloxacino con metronidazol para su confirmación. El análisis de costos demostró una reducción del coste global del $82.8 \%$ y de 1985 euros por paciente.

\section{Financiación}

Los autores declaran que no recibieron financiación para esta publicación.

\section{Conflicto de intereses}

Los autores declaran que no tienen ningún conflicto de intereses para el desarrollo y la publicación del trabajo.

\section{Responsabilidades éticas}

Protección de personas y animales. Los autores declaran que para esta investigación no se han realizado experimentos en seres humanos ni en animales.

Confidencialidad de los datos. Los autores declaran que han seguido los protocolos de su centro de trabajo sobre la publicación de datos de pacientes.

Derecho a la privacidad y consentimiento informado. Los autores han obtenido el consentimiento informado de los pacientes y/o sujetos referidos en el artículo. Este documento obra en poder del autor de correspondencia.

\section{Bibliografía}

1. Tursi A. Efficacy, safety and applicability of outpatient treatment for diverticulitis. Drug Healthc Patient Saf. 2014;6:29-36.

2. Bharucha AE, Parthasarathy G, Ditah I, Fletcher JG, Ewelukwa O, Pendlimari $\mathrm{R}$, et al. Temporal trends in the incidence and natural history of diverticulitis: a population-based study. Am J Gastroenterol. 2015;110:1589-96.
3. Balasubramanian I, Fleming C, Mohan HM, Schmidt K, Haglind E, Winter DC. Out-patient management of mild or uncomplicated diverticulitis: a systematic review. Dig Surg. 2017;34:151-60.

4. Sánchez-Velázquez P, Grande L, Pera M. Outpatient treatment of uncomplicated diverticulitis: a systematic review. Eur J Gastroenterol Hepatol. 2016;28:622-7.

5. Moya P, Bellon M, Arroyo A, Galindo I, Candela F, Lacueva J, et al. Outpatient treatment in uncomplicated acute diverticulitis: 5-year experience. Turk J Gastroenterol. 2016;27:330-5.

6. Jackson JD, Hammond T. Systematic review: outpatient management of acute uncomplicated diverticulitis. Int J Colorectal Dis. 2014;29:775-81.

7. Biondo S, Golda T, Kreisler E, Espin E, Vallribera F, Oteiza F, et al. Outpatient versus hospitalization management for uncomplicated diverticulitis: a prospective multicenter, randomized clinical trial (DIVER Trial). Ann Surg. 2014;259:38-44.

8. Lorente L, Corts F, Alonso S, Pascual M, Salvans S, Courtier R, et al. Outpatient treatment of uncomplicated acute diverticulitis: impact on healthcare costs. Cir Esp. 2013;91:504-9.

9. Moya P, Arroyo A, Pérez-Legaz J, Serrano P, Candela F, Soriano-Irigaray $L$, et al. Applicability, safety and efficiency of outpatient treatment in uncomplicated diverticulitis. Tech Coloproctol. 2012;16:301-7.

10. Martín Gil J, Serralta De Colsa D, García Marín A, Vaquero Rodríguez A, Rey Valcárcel C, Pérez Díaz MD, et al. Safety and efficiency of ambulatory treatment of acute diverticulitis. Gastroenterol Hepatol. 2009;32:83-7

11. Peláez N, Pera M, Courtier R, Sánchez J, Gil MJ, Parés D, et al. Applicability, safety and efficacy of an ambulatory treatment protocol in patients with uncomplicated acute diverticulitis. Cir Esp. 2006;80:369-72.

12. Mizuki A, Nagata $H$, Tatemichi M, Kaneda S, Tsukada N, Ishii H, et al. The out-patient management of patients with acute mild-to-moderate colonic diverticulitis. Aliment Pharmacol Ther. 2005;21:889-97.

13. Ambrosetti $P$, Jenny A, Becker C, Terrier T, Morel P. Acute left colonic diverticulitis-compared performance of computed tomography and watersoluble contrast enema: prospective evaluation of 420 patients. Dis CoIon Rectum. 2000;43:1363-7.

14. Bone RC, Balk RA, Cerra FB, Dellinger RP, Fein AM, Knaus WA, et al. Definitions for sepsis and organ failure and guidelines for the use of innovative therapies in sepsis. The ACCP/SCCM Consensus Conference Committee. American College of Chest Physicians/Society of Critical Care Medicine. Chest. 1992;101:1644-55.

15. Decreto Legislativo del Consell de la Generalitat, por el que se aprueba el Texto Refundido de la Ley de Tasas de la Generalitat. Decreto Legislativo $1 / 2005$ de 25 de febrero. Diario Oficial de la Comunidad Valenciana, n. 4971 (22-03-2005).

16. Ridgway PF, Latif A, Shabbir J, Ofriokuma F, Hurley MJ, Evoy D, et al. Randomized controlled trial of oral versus intravenous therapy for the clinically diagnosed acute uncomplicated diverticulitis. Colorectal Dis. 2009;11:941-6.

17. Rodríguez-Cerrillo M, Poza-Montoro A, Fernández-Díaz E, Romero-Al. Patients with uncomplicated diverticulitis and comorbidity can be treated at home. Eur J Intern Med. 2010;21:553-4.

18. Joliat GR, Emery J, Demartines N, Hübner M, Yersin B, Hahnloser D. Antibiotic treatment for uncomplicated and mild complicated diverticulitis: outpatient treatment for everyone. Int J Colorectal Dis. 2017; 32:1313-9.

19. de Korte N, Kuyvenhoven JP, van der Peet DL, Felt-Bersma RJ, Cuesta MA, Stockmann HB. Mild colonic diverticulitis can be treated without antibiotics. A case-control study. Colorectal Dis. 2012;14:325-30.

20. Chabok A, Pahlman L, Hiern F, Haapaniemi S, Smedh K. Randomized clinical trial of antibiotics in acute uncomplicated diverticulitis. Br J Surg. 2012;99:532-9.

21. Ünlu Ç, Gunadi PM, Gerhards MF, Boermeester MA, Vrouenraets BC. Outpatient treatment for acute uncomplicated diverticulitis. Eur J Gastroenterol Hepatol. 2013;25:1038-43.

22. Estrada Ferrer O, Ruiz Edo N, Hidalgo Grau LA, Abadal Prades M, Del Bas Rubia M, García Torralbo EM, et al. Selective non-antibiotic treatment in sigmoid diverticulitis: is it time to change the traditional approach? Tech Coloproctol. 2016;20:309-15.

23. Daniels L, Ünlü Ç, de Korte N, van Dieren S, Stockmann HB, Vrouenraets BC, et al. Randomized clinical trial of observational versus antibiotic treatment for a first episode of CT-proven uncomplicated acute diverticulitis. Br J Surg. 2017;104:52-61.

24. Schug-Pass $C$, Geers $P$, Hügel $O$, Lippert H, Köckerling F. Prospective randomized trial comparing short-term antibiotic therapy versus standard therapy for acute uncomplicated sigmoid diverticulitis. Int $\mathrm{J}$ Colorectal Dis. 2010;25:751-9.

25. Etzioni DA, Chiu VY, Cannom RR, Burchette RJ, Haigh PI, Abbas MA. Outpatient treatment of acute diverticulitis: rates and predictors of failure. Dis Colon Rectum. 2010;53:861-5.

26. Park HC, Kim BS, Lee BH. Management of right colonic uncomplicated diverticulitis: outpatient versus inpatient management. World J Surg. 2011;35:1118-22. 